\title{
ARTICLE
}

Received 4 Nov 2014 | Accepted 19 Feb 2015 | Published 26 Mar $2015 \quad$ DOl: 10.1038/ncomms7675

\section{A megacomplex composed of both photosystem reaction centres in higher plants}

M. Yokono ${ }^{1,2}$, A. Takabayashi ${ }^{1,2}$, S. Akimoto ${ }^{3,4} \&$ A. Tanaka $a^{1,2}$

Throughout the history of oxygen evolution, two types of photosystem reaction centres (PSI and PSII) have worked in a coordinated manner. The oxygen evolving centre is an integral part of PSII, and extracts an electron from water. PSI accepts the electron, and accumulates reducing power. Traditionally, PSI and PSII are thought to be spatially dispersed. Here, we show that about half of PSIls are physically connected to PSIs in Arabidopsis thaliana. In the PSI-PSII complex, excitation energy is transferred efficiently between the two closely interacting reaction centres. PSII diverts excitation energy to PSI when PSII becomes closed-state in the PSI-PSII complex. The formation of PSI-PSII complexes is regulated by light conditions. Quenching of excess energy by PSI might be one of the physiological functions of PSI-PSII complexes.

\footnotetext{
${ }^{1}$ Institute of Low Temperature Science, Hokkaido University, Sapporo 060-0819, Japan. ${ }^{2}$ CREST, JST, Sapporo 060-0819, Japan. ${ }^{3}$ Molecular Photoscience Research Center, Kobe University, Kobe 657-8501, Japan. ${ }^{4}$ CREST, JST, Kobe 657-8501, Japan. Correspondence and requests for materials should be addressed to M.Y. (email: filia@mac.com) or to S.A. (email: akimoto@hawk.kobe-u.ac.jp).
} 
$\mathrm{n}$ oxygenic photosynthetic organisms, two photosystem reaction centres (PSI and PSII) cooperate to split water ${ }^{1}$. The regulation of photosynthesis has been studied based on the idea that all photosystems exist separately in thylakoid membranes. However, recent reports have questioned this concept. A megacomplex consisting of PSII, PSI and phycobilisomes was identified in cyanobacteria, in which phycobilisomes supply excitation energy to both photosystems ${ }^{2}$. Although the distribution of the two reaction centres is inhomogeneous in higher plants, PSI and PSII still have a chance to directly interact. The two reaction centres coexist in a flexible nonappressed region ${ }^{3,4}$, and about half of PSIIs exist in stroma thylakoids and grana margins where PSIs also exist ${ }^{5}$. Recently, Järvi et $a l^{6}{ }^{6}$ reported coexistence of PSI and PSII complexes together with light-harvesting complexes (LHCs) in large-pore blue-native polyacrylamide gel electrophoresis (PAGE) from Arabidopsis, suggesting possible interaction between the complexes. In this study, we combined large-pore clear-native PAGE (lpCN-PAGE) and time-resolved spectroscopy to examine whether PSI and PSII interact with each other in Arabidopsis thaliana.

Here, we show that about half of PSIIs are physically connected to PSIs in Arabidopsis thaliana. In PSI-PSII complexes isolated by lpCN-PAGE, excitation energy is transferred efficiently between the two closely interacting reaction centres. PSII diverts excitation energy to PSI when PSII becomes closed-state in the PSI-PSII complex. It may help to protect PSII from excess energy, and keep a balance between two photosystems. The formation of PSI-PSII complexes is regulated by light conditions.

\section{Results and Discussion}

Energy transfer from the PSII reaction center to PSI in leaves. First, we examined whether PSI-PSII complexes existed in A. thaliana by delayed fluorescence analysis. Delayed fluorescence with a time constant of $\sim 20 \mathrm{~ns}$ at $-196^{\circ} \mathrm{C}$ is derived from charge recombination in PSII ${ }^{7,8}$. PSI and LHCII by themselves did not show delayed fluorescence in this timescale at this temperature (Supplementary Figs 1 and 2), consistent with previous reports ${ }^{9-11}$. Therefore, the delayed fluorescence spectrum reflects the excitation distribution after direct excitation of PSII ${ }^{12}$. If PSI-PSII complexes do not exist, PSII does not transfer excitation energy to PSI, and the excitation energy generated by the charge recombination at PSII is distributed only in PSII even though the two reaction centres exist in the same place. On the other hand, in the case that PSIPSII complexes exist, the energy is also distributed to PSI. Therefore, delayed fluorescence should appear in the PSI wavelength region at $-196^{\circ} \mathrm{C}$ if PSI-PSII complexes exist ${ }^{12}$.

Figure 1a shows the delayed fluorescence spectra of an intact leaf of the wild type measured at $-196^{\circ} \mathrm{C}$. The delayed fluorescence spectrum showed a large peak in the PSI wavelength region $(\sim 735 \mathrm{~nm})$ and a peak in the PSII wavelength region $(\sim 690 \mathrm{~nm})$, indicating the existence of PSI-PSII complexes. After correction to remove the effects of differences in fluorescence quantum yield among chlorophyll forms (Supplementary Fig. 3f $)^{12}$, we found that $48 \%$ of the excitation energy generated by charge recombination at PSII was transferred to PSI. Interestingly, this value was similar to the ratio of the relative distribution of PSII core proteins in unstacked regions (stroma thylakoids and grana margins) where PSI exists ${ }^{5}$. On the basis of the delayed fluorescence intensity, about $74 \%$ of PSI was calculated to be bound to PSII (Supplementary Note 1). We also measured delayed fluorescence with a chlorophyll $b$-less mutant (ch1-1), in which the LHCII level is drastically decreased, and five lhc mutants (lhcal-4 and lhcb5) to check whether LHCs acted as linkers between PSI and PSII. All mutants showed a large PSI peak in their delayed fluorescence spectra (Supplementary Fig. 4, 6th lifetime component). These results suggested that LHCs were not required for PSI-PSII complex formation.
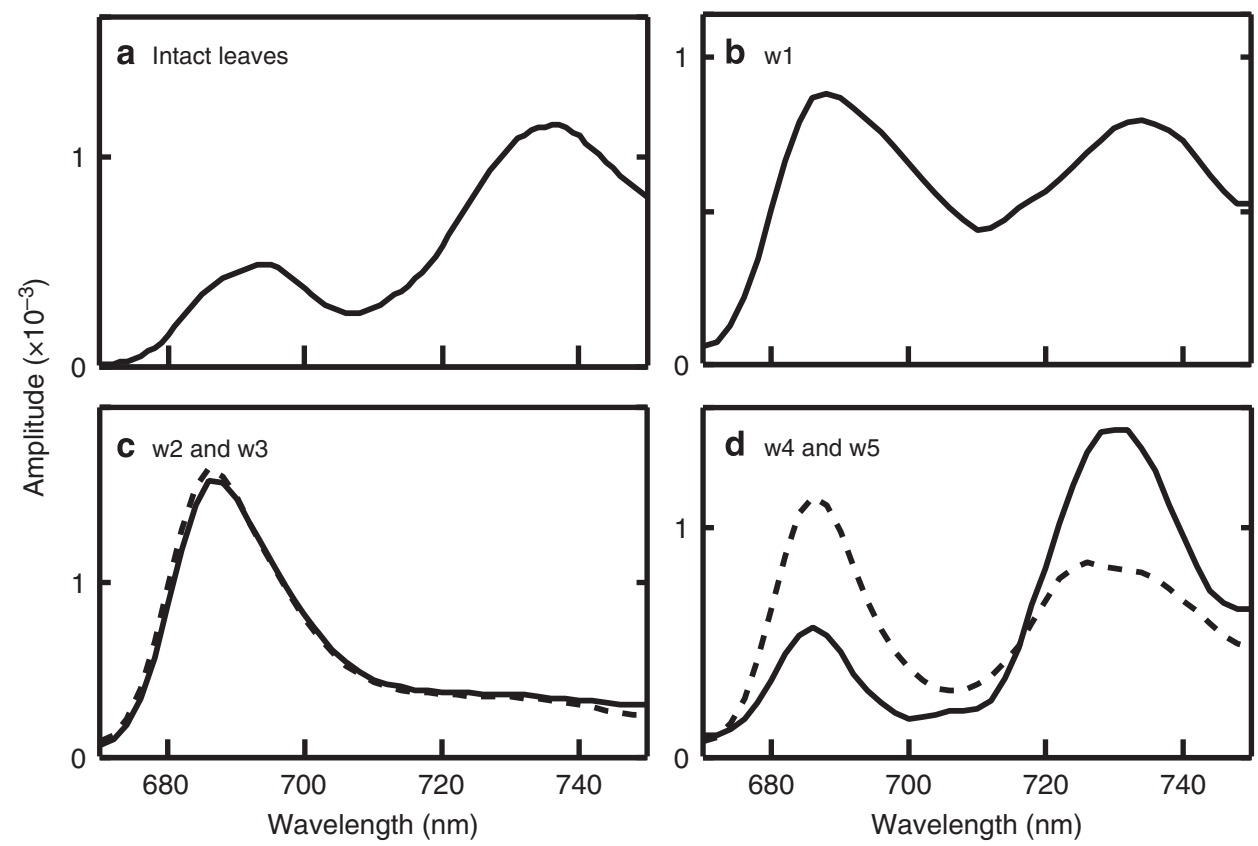

Figure 1 | Delayed fluorescence spectra obtained at $-196^{\circ} \mathbf{C}$. Other lifetime components are shown in Supplementary Fig. 3. (a) Intact leaf of the wild type. (b-d) The w1-w5 samples from IpCN-PAGE gel pieces corresponding to the regions described in Fig. 2. (b) The w1 sample. Decay curves were measured two times with a 1.2-ps interval and five times with a 24.4-ps interval. (c) The w2 (black solid line) and w3 (black dotted line) samples. Decay curves were measured two times with 1.2-ps and 24.4-ps intervals. (d) The w4 (black solid line) and w5 (black dotted line) samples. Decay curves were measured two times with 1.2-ps and 24.4-ps intervals. Excitation energy transfer from PSII to PSI was observed in the w1, w4 and w5 samples. 
Isolation of PSI-PSII complexes by lpCN-PAGE. Next, we isolated thylakoid membranes from the wild type, and separated the complexes by lpCN-PAGE. We observed that $26 \%$ of the excitation energy in PSII was transferred to PSI in the thylakoid membrane (Supplementary Fig. 3e,f). The detergent digitonin partially solubilized thylakoid membranes. The insoluble fraction contained $\sim 60 \%$ of the chlorophyll and was composed of PSIILHCII complexes; little PSI fluorescence was detected (Supplementary Fig. 5). This indicated that the insoluble fraction was mainly composed of grana stack regions. The soluble fraction was subjected to large-pore gel electrophoresis.

All soluble fractions passed through the stacking gel, indicating that the complexes were completely solubilized in the sample (also see Supplementary Fig. 6). The electrophoretic profile of the wild type was very similar to that in a previous report (Fig. 2) ${ }^{6}$. The largest band was estimated as $>2,400 \mathrm{kDa}$, and contained PSI, PSII, LHCII and LHCI revealed by silver staining, immunoblot analysis and liquid chromatography-mass spectrometry (LCMS)/MS analysis (Supplementary Figs $7-9)^{6,13}$. We called this green area the w1 sample. According to the estimate of protein amounts from LC-MS/MS data by the exponentially modified protein abundance index method, PSI, PSII, LHCII and LHCI subunits made up $94 \%$ in the w1 sample (Supplementary Fig. 9). The second and third areas, referred to as w2 and w3 samples, respectively, were larger than $720 \mathrm{kDa}$ (Fig. 2), and assigned to PSII-LHCII complexes and comigrated PSI oligomers (Supplementary Figs $7-10)^{6,13}$. In the range of $480-720 \mathrm{kDa}$ (Fig. 2), there was a broad green area that contained two distinct bands (thin broken arrows) and a faint broad green band (thin solid arrow). The heavier band was assigned to PSI-LHCI/II, and the lighter band was assigned to PSI-LHCI and comigrated PSII complexes, ${ }^{6,13}$. lpCN/SDS-PAGE and immunoblot analyses revealed that the additional band indicated

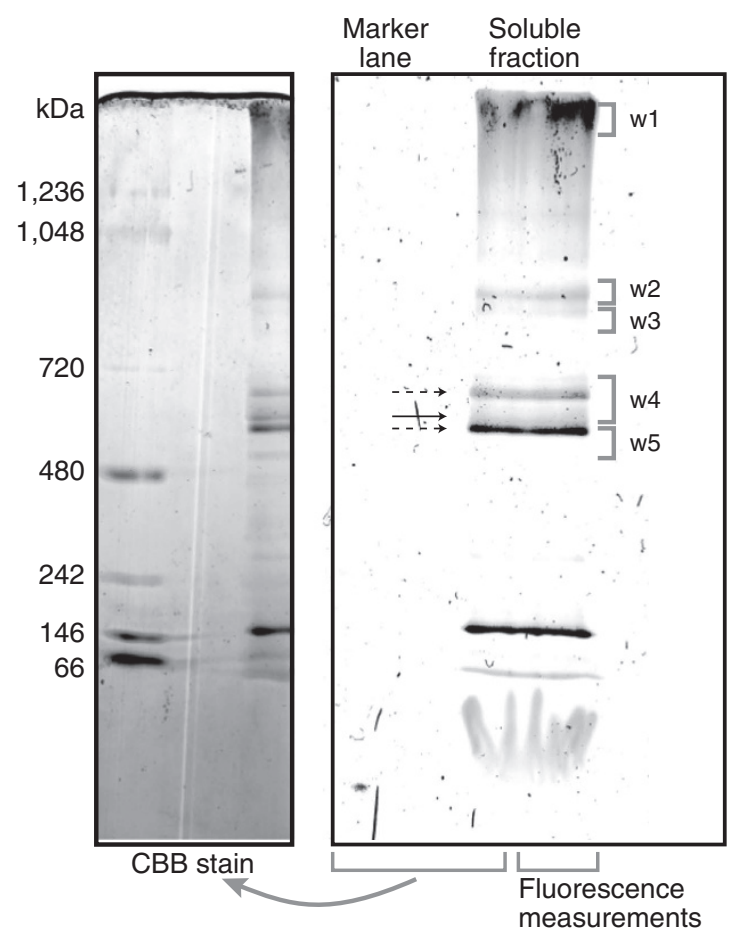

Figure 2 | IpCN-PAGE analysis of thylakoid membranes of the wild type solubilized by $1 \%$ digitonin. The marker lane and the left edge of sample lane were cut off and stained with Coomassie Brilliant Blue. The remaining sample lane was used for fluorescence measurements. by the thin solid arrow in Fig. 2 contained both PSI and PSII (Supplementary Figs 7 and $8 \mathrm{a}$ ). The lpCN-PAGE gel exhibited a green colour between the two distinct bands, indicating the presence of another complex(es) in this region. We divided this region into two samples, w4 and w5; the w4 sample contained the upper band and the faint broad band (Fig. 2).

Delayed fluorescence of isolated PSI-PSII complexes. Then, we measured the delayed fluorescence spectra of these samples at $-196^{\circ} \mathrm{C}$. In the w1 sample, the delayed fluorescence spectrum (18 ns) showed clear peaks in both the PSII and PSI wavelength regions, indicating excitation energy transfer from PSII to PSI (Fig. 1b). In the PSI wavelength region, the peak position was $735 \mathrm{~nm}$, which was derived from low-energy chlorophylls in PSI$\mathrm{LHCI}^{14,15}$. This result was consistent with the PAGE analysis and LC-MS/MS analysis (Supplementary Figs 7-9), which showed the existence of a megacomplex composed of PSI, PSII, LHCI and LHCII in A. thaliana ${ }^{6}$. In the w1 sample, $26 \%$ of the excitation energy generated by charge recombination at PSII was transferred to PSI-LHCI at $-196^{\circ} \mathrm{C}$ (Supplementary Fig. 3f). Fluorescence originating from LHC antennae $(\sim 680 \mathrm{~nm})$ decreased within 100 ps in the w1 sample (Supplementary Fig. 3b, 1st component), indicating efficient energy transfer from LHCI and LHCII to the reaction centres. Hereafter, we call this megacomplex in the w1 sample the 'larger PSI-PSII complex'. The w1 sample became more intense under milder solubilization, but the spectroscopic properties were not altered (Supplementary Fig. 11).

The delayed fluorescence spectra of the w2 and w3 samples (Fig. 1c) showed only a peak at $686 \mathrm{~nm}$ that was derived from PSII, despite the presence of a lot of PSI in these samples, reflecting a lack of energy transfer from PSII to PSI. This result clearly indicated that w2 and w3 did not contain PSI-PSII complexes.

The delayed fluorescence spectra of the w4 and w5 samples showed clear peaks in both the PSII and PSI wavelength regions (Fig. 1d). These results indicated that $\mathrm{w} 4$ and $\mathrm{w} 5$ contained not only PSI-LHCI/II, PSI-LHCI and PSII dimers, but also a smaller PSI-PSII complex. At least $76 \%$ and $45 \%$ of the excitation energy generated by charge recombination at PSII was transferred to PSI in w4 and w5 at $-196^{\circ} \mathrm{C}$, respectively (Supplementary Fig. 3f). The smaller PSI-PSII complex in w4 and w5 contained fewer LHCIIs (Supplementary Figs 7-9). We assumed that the smaller PSI-PSII complex contained a PSI core and PSII monomer, while LHCII was bound to PSII and PSI peripherally in the larger PSI-PSII complex (Supplementary Note 2; Supplementary Fig. 12). According to the blue-shifted PSI peak in the delayed fluorescence spectrum (Fig. 1d), some small subunits including Lhca might be lacking in the smaller PSI-PSII complex ${ }^{16,17}$.

We found two types of PSI-PSII complexes, larger and smaller complexes. In the larger PSI-PSII complex, rapid energy migration and trapping occurred at room temperature. Figure 3 shows the fluorescence decay-associated spectra of the w1 sample (the larger PSI-PSII complex) at $20^{\circ} \mathrm{C}$. The 20 -ps lifetime component showed a positive peak around $690 \mathrm{~nm}$ and a negative peak around $730 \mathrm{~nm}$, reflecting energy transfer between PSII and PSI. The 100-ps lifetime component showed the largest amplitude, with two broad peaks around $690 \mathrm{~nm}$ and $720 \mathrm{~nm}$. Excitation energy was distributed to both PSII and PSI and trapped by P680 and P700 within 100 ps after antenna excitation. The ratio of these two peaks was about 2:3, reflecting the trapping ratio of P680 and P700 in the larger PSI-PSII complex after antenna excitation. According to reduced-minus-oxidized absorption analysis of cytochrome 6559 and P700 in the w1 sample, the ratio of PSI to PSII was close to 1:1 in the w1 sample (Supplementary Table 1). These results indicate that PSI accepts a 


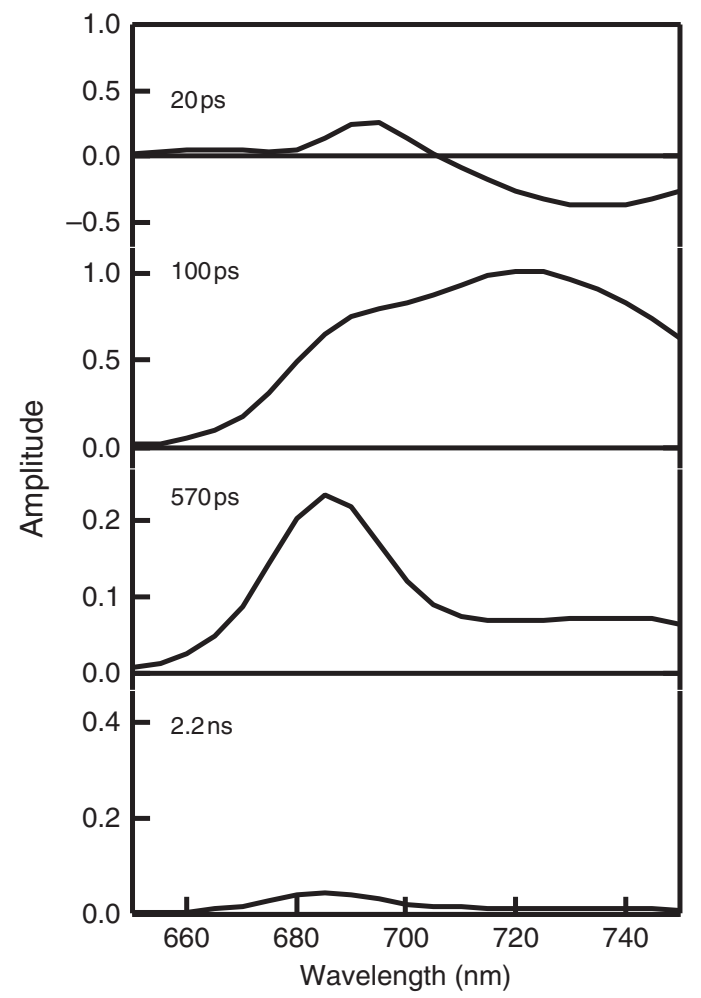

Figure 3 | Fluorescence decay-associated spectra of the w1 sample

$\left(20^{\circ} \mathbf{C}\right)$. Decay curves were measured two times with a 2.4 -ps interval. Lifetime components were normalized to the lifetime component of the largest amplitude in the sample.

slightly larger amount of excitation energy than PSII in the larger PSI-PSII complex when the PSII reaction center is open. The 570-ps and 2.2-ns components showed a peak at $685 \mathrm{~nm}$, reflecting trapped excitation energy at PSII that was not transferred to PSI. Some PSII might energetically detach from PSI during isolation procedures. The wl sample showed fast fluorescence decay even in the presence of (3-(3,4-dichlorophenyl)-1,1-dimethylurea) (Supplementary Fig. 13), which indicates that PSII can divert excitation energy to PSI when PSII becomes closed-state in the PSI-PSII complex. The rapid energy migration disappeared when the w1 sample was incubated in $1 \% \alpha$-dodecyl maltoside (Supplementary Fig. 14; Supplementary Note 3), indicating detachment of PSII and LHCII from PSI.

Dynamic response of the PSI-PSII complex to light intensity. To elucidate the physiological function of the PSI-PSII complex, we examined the changes in PSI-PSII complexes when the plants were exposed to different light conditions. Intact leaves were corrected under various light conditions, and fluorescence decayassociated spectra were constructed to obtain delayed fluorescence spectra (Supplementary Figs 15,16). After a 14-h dark period, the estimated ratio of PSI-PSII complexes was $46 \%$ (Fig. 4a, dark). When the plants were exposed to growth light $\left(50 \mu \mathrm{mol}\right.$ photons $\left.\mathrm{m}^{-2} \mathrm{~s}^{-1}\right)$ for $3 \mathrm{~h}$, the ratio increased to $63 \%$ (Fig. 4a, A). High-light treatment $\left(2,000 \mu \mathrm{mol}\right.$ photons $\left.\mathrm{m}^{-2} \mathrm{~s}^{-1}\right)$ resulted in further elevation to $70 \%$ (Fig. $4 \mathrm{a}, \mathrm{D}$ ). These results indicate that an increase in light intensity is accompanied by an increase in PSI-PSII complexes. In contrast, when the light intensity was lowered to $50 \mu \mathrm{mol}$ photons $\mathrm{m}^{-2} \mathrm{~s}^{-1}$, the ratio of PSI-PSII complexes decreased to 55\% within 1 min (Fig. 4a, E). This indicates that energetic decoupling of PSII from PSI occurs immediately after transfer from high-light to growth-light

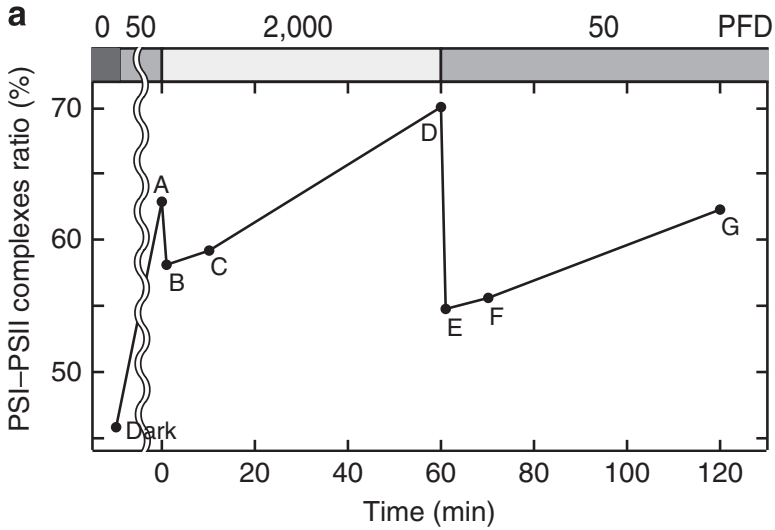

b

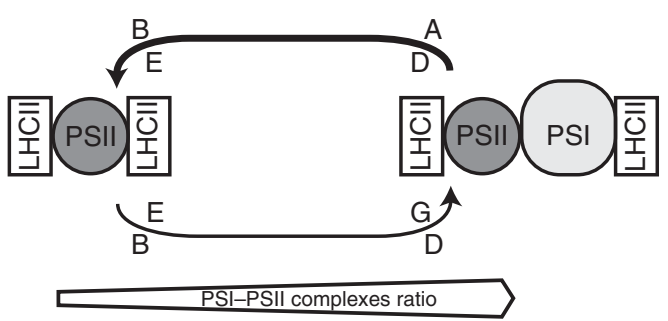

Figure 4 | Change of the PSI-PSII complexes ratio in the wild-type leaf depending on light conditions. (a) The ratios were determined from the delayed fluorescence spectra and mean lifetimes shown in Supplementary Fig. 15 as described in Supplementary Fig. 3 and a previous report ${ }^{12}$. Sample details are shown in Supplementary Fig. 16. Decay curves were measured one time and two times with 1.2-ps interval and 24.4-ps interval, respectively. (b) Schematic representation of the megacomplex formation. The thin arrow represents slow stages such as PSII migration from the grana stack and/or repair processes. The thick arrow represents the fast stage of energetic decoupling of PSII from PSI depending on sudden light changes.

conditions. Then, the ratio gradually recovered to $62 \%$ over $1 \mathrm{~h}$ (Fig. 4a, G), which reflects reformation of PSI-PSII complexes.

It has been suggested that phosphorylation of LHC and PSII core components is involved in the migration of photosystems between grana stack and stroma thylakoid regions ${ }^{18}$. To investigate the role of phosphorylation of these proteins, we examined the delayed fluorescence of mutants defective in the phosphorylation of individual proteins. Although some perturbations were observed, three mutants $(\sin 7, \sin 8$ and tap38) related to phosphorylation of the photosystems possessed PSI-PSII complexes (Supplementary Fig. 17a-c, 6th components) ${ }^{19}$, indicating that phosphorylated LHCII, D1 and D2 are not required for PSI-PSII complex formation.

In this study, we elucidated that the PSII-originated delayed fluorescence from PSI is derived from a PSI-PSII complex. Although the molecular structure of the PSI-PSII complex was not clarified in this study, the core antenna of both photosystems might associate directly because mutants deficient in LHCIIs or LHCIs showed the same delayed fluorescence spectra as the wild type. PSII-originated delayed fluorescence from PSI has been observed from various organisms including cyanobacteria and red algae. Our results suggest that PSI-PSII complexes play important roles in a wide range of photosynthetic organisms, not only in red algae $^{12,20}$ but also in higher plants. We found that excitation energy migrates between PSI and PSII within $100 \mathrm{ps}$, and that all of the excitation energy at PSII can be potentially transferred to PSI when PSII is closed-state in the complex. This suggests that PSI can quench PSII when PSII is excessively excited. This idea is 
supported by the observation that PSI-PSII complexes increased under high-light conditions. Quenching of excess energy by PSI might be one of the physiological functions of PSI-PSII complexes.

\section{Methods}

Plant materials. The Columbia ecotype of wild-type and mutant $A$. thaliana were grown at $22^{\circ} \mathrm{C}$ under short-day conditions $\left(8 \mathrm{~h}\right.$ light, $50 \mu \mathrm{mol}$ photons $\mathrm{m}^{-2} \mathrm{~s}^{-1}$, white fluorescent lamp) in trays $(\sim 5 \mathrm{~cm}$ depth) containing layers of horticultural soil (National Federation of Agricultural Cooperative Association, Japan) and expanded vermiculite (Taiheiyo Coal Services and Transportation, Japan). The fertilizer ratio in this mixture was $\mathrm{N}: \mathrm{P}_{2} \mathrm{O}_{5}: \mathrm{K}_{2} \mathrm{O}: \mathrm{MgO}=34: 135: 22: 15$. Leaves were harvested about 9 weeks after seeding. Mutant seeds were obtained from the Arabidopsis Biological Resource Center.

Sample separation using IpCN-PAGE. Thylakoid membranes were isolated and solubilized with $1 \%$ digitonin (Sigma-Aldrich Japan, Tokyo) at $21^{\circ} \mathrm{C}$ as described previously $^{6}$. In brief, leaves were harvested from 80 individual plants. One leaf was used for spectroscopic measurements, and the remaining leaves were used for thylakoid isolation. Leaves were ground in the grinding buffer $(50 \mathrm{mM}$ Hepes $/ \mathrm{KOH}$ pH 7.5, $330 \mathrm{mM}$ sorbitol, $2 \mathrm{mM}$ EDTA, $1 \mathrm{mM} \mathrm{MgCl} 2,5 \mathrm{mM}$ ascorbate, $0.05 \%$ bovine serum albumin, $10 \mathrm{mM}$ sodium fluoride) at $4{ }^{\circ} \mathrm{C}$ and the homogenate was filtered through Miracloth layers. After centrifugation $\left(5,000 \mathrm{~g}, 4 \mathrm{~min}, 4^{\circ} \mathrm{C}\right)$, the pellet was suspended in the shock buffer $(50 \mathrm{mM}$ Hepes $/ \mathrm{KOH}$ pH 7.5, $5 \mathrm{mM}$ sorbitol, $5 \mathrm{mM} \mathrm{MgCl} 2,10 \mathrm{mM}$ sodium fluoride) at $4{ }^{\circ} \mathrm{C}$ and the suspension was centrifuged again. The thylakoid pellet was suspended in the storage buffer $(50 \mathrm{mM}$ Hepes/KOH pH 7.5, $100 \mathrm{mM}$ sorbitol, $10 \mathrm{mM} \mathrm{MgCl}, 10 \mathrm{mM}$ sodium fluoride) to a chlorophyll concentration of $1.5 \mathrm{mg} \mathrm{ml}^{-1}$. The thylakoid membrane was solubilized with $1 \%$ digitonin at $0.5 \mathrm{mg}$ chlorophyll per $\mathrm{ml}$ in the $25 \mathrm{BTH} 20 \mathrm{G}$ buffer $(25 \mathrm{mM}$ BisTris/HCl pH 7.0, 20\% (w/v) glycerol, $0.25 \mathrm{mg} \mathrm{ml}^{-1}$ Pefabloc SC, $10 \mathrm{mM}$ sodium fluoride) at $21{ }^{\circ} \mathrm{C}$. lpCN-PAGE was performed essentially as described previously ${ }^{6}$. In brief, in separation gel, acrylamide gradient was $3.5-12.5 \%$, where the ratio of bisacrylamide to the total acrylamide was $3 \%$. In stacking gel, total acrylamide concentration was $3 \%$, where the ratio of bisacrylamide to the total acrylamide was $20 \%$. Both gels were prepared using the gel buffer containing $0.5 \mathrm{M} 6$-aminocaproic acid and $50 \mathrm{mM}$ BisTris/ $\mathrm{HCl} \mathrm{pH} 7.0$ at $4^{\circ} \mathrm{C}$. Anode buffer contained $50 \mathrm{mM}$ BisTris/ $/ \mathrm{HCl} \mathrm{pH} 7.0$ at $4{ }^{\circ} \mathrm{C}$. The only difference from the previous study was that the cathode buffer $\left(50 \mathrm{mM}\right.$ Tricine, $15 \mathrm{mM}$ BisTris/ $\mathrm{HCl} \mathrm{pH} 7.0$ at $4{ }^{\circ} \mathrm{C}, 0.05 \%$ sodium deoxycholate) did not contain Coomassie Brilliant Blue or dodecyl maltoside because the former acted as a competitive quencher of chlorophyll fluorescence in LHCII, and the latter deteriorated the megacomplex (Supplementary Fig. 18). Note that the stacking gel was polymerized for $40 \mathrm{~min}$ above $26^{\circ} \mathrm{C}$. Gel images were captured with a scanner (GT-X970, Epson, Japan), and input levels were adjusted by curve adjustment (Photoshop CS5.1, Adobe Systems, USA). Typically, a black point was set to a default value, and a white point was set to $\sim 200$. Intact leaves and bands in lpCN-PAGE were excised and put into quartz tubes of 5-mm diameter under dim-light conditions and stored at $-80^{\circ} \mathrm{C}$ prior to spectroscopic measurements. Intact leaves for the varying growth light experiment (Supplementary Fig. 16) were excised under each growth light condition and frozen within $10 \mathrm{~s}$. SDS-PAGE and immunoblot analyses were performed as described previously ${ }^{21}$. In brief, the excised lanes of lpCN-PAGE were immersed in a solution containing $1 \%$ SDS and $1 \% 2$-mercaptoethanol at $25^{\circ} \mathrm{C}$ for $1 \mathrm{~h}$ and run on polyacrylamide gels using Laemmli buffer. Total acrylamide concentration was $14 \%$, where the ratio of bisacrylamide to the total acrylamide was $2.6 \%$. The gels were prepared using the gel buffer containing $12.4 \mathrm{mM}$ Tris $/ \mathrm{HCl} \mathrm{pH} 6.8$ and $0.13 \%$ SDS. After electrophoresis, a gel was stained using the silver stain kit (Pierce Silver Stain for Mass Spectrometry, Thermo Scientific, USA) and the remaining gels were used for immunoblot analysis. The proteins were blotted onto polyvinylidene fluoride membranes with transfer buffer ( $25 \mathrm{mM}$ Tris, $192 \mathrm{mM}$ glycine, $20 \%$ methanol). The protein were detected using the Western Lightning Chemiluminescence Reagent Plus (Perkin Elmer, Life Sciences). Antibody dilutions were as follows: PsaA/B (raised against purified $\mathrm{PsaA} / \mathrm{B}^{21}, 1: 50,000$ ), $\mathrm{CP} 47$ (Agrisera AS04 038, 1:3,000), CP43 (Agrisera AS11 1787, 1:3,000), D1 (Agrisera AS01 016, 1:9,000), Lhcb1 (Agrisera AS01 004, 1:5,000), Lhcb2 (Agrisera AS01 003, 1:5,000), Lhcb3 (Agrisera AS01 002, 1:1,000), Lhca3 (Agrisera AS01 007, 1:5,000), Lhca4 (Agrisera AS01 008, 1:5,000), Cyt $b_{6}$ (Agrisera AS03 034, 1:15,000).

Spectroscopic analyses. Time-resolved spectroscopic measurements and analysis were performed as described previously but using a 5 -nm optical slit width ${ }^{22}$. The excitation wavelength was $425 \mathrm{~nm}$ and the repetition rate was $2.9 \mathrm{MHz}$, which did not interfere with measurements up to $100 \mathrm{~ns}$ ( $24.4 \mathrm{ps}$ per channel $\times 4,096$ channels). To improve time resolution, time-resolved fluorescence was also measured up to $5 \mathrm{~ns}(1.2 \mathrm{ps}$ per channel $\times 4,096$ channels $)$ at $-196^{\circ} \mathrm{C}$. At $20^{\circ} \mathrm{C}$, time-resolved fluorescence was measured up to $10 \mathrm{~ns}(2.4 \mathrm{ps}$ per channel $\times 4,096$ channels). The excitation laser intensity was $\sim 0.1 \mathrm{~nJ}$ per pulse or less. All experiments were replicated at least two times. Reproducibility of decay curves were verified, and the curves were summed and kinetics were analysed. All samples were measured using the surface-reflection technique (Supplementary Fig. 19) ${ }^{23}$.
In brief, fluorescence was corrected at a excitation point on surface of the sample via a narrow slit $(0.5 \mathrm{~mm}$ width). The excitation and collection position on the sample was adjusted using guide laser and optical stages. In this experimental condition, reabsorption does not affect delayed fluorescence in the PSI wavelength region (Supplementary Fig. 20). On the basis of changes of $\chi^{2}$ and differences between decay curves and fitting curves ${ }^{22}$, we chose six-component analysis for the $-196{ }^{\circ} \mathrm{C}$ measurement results, and four-component analysis for the $20^{\circ} \mathrm{C}$ measurement results. Final time resolution was about 10 ps. Steady-state fluorescence spectra were measured using an F-2500 spectrophotometer (Hitachi). The optical slit widths for excitation and emission were 10 and $2.5 \mathrm{~nm}$, respectively.

For reduced-minus-oxidized absorption measurement ${ }^{24}$, about $3 \mathrm{ml}$ of each excised band from lpCN-PAGE was immersed in the same volume of 25BTH20G buffer ${ }^{6}$ with $2 \% \beta$-dodecyl maltoside for $24 \mathrm{~h}\left(4^{\circ} \mathrm{C}\right.$, dark). The extracted solution was dispensed in 1.5-ml aliquots and used for quantitative analysis of cytochrome b559 and P700 in the excised band. Spectra were recorded using a U-3310 spectrophotometer (Hitachi) at room temperature. The optical slit width was $5 \mathrm{~nm}$, and the scan speed was $120 \mathrm{~nm} \mathrm{~min}^{-1}$. Hydroquinone and potassium hexacyanoferrate (III) obtained from Nacalai Tesque (Japan) were used as oxidants of cytochrome 6559 and P700, respectively. Sodium L-ascorbate was added as a reducing agent. For P700, an extinction coefficient of $64 \mathrm{mM}^{-1} \mathrm{~cm}^{-1}$ was used For cytochrome b559, an extinction coefficient of $25 \mathrm{mM}^{-1} \mathrm{~cm}^{-1}$ was used ${ }^{26}$. The chlorophyll concentration and $a / b$ ratio were determined as described previously ${ }^{27}$ In brief, the extracted solution was diluted with $80 \%$ acetone, and centrifuged at $18,000 \mathrm{~g}$ for $1 \mathrm{~min}$. Absorbance of the supernatant was measured at 646.6 and $663.6 \mathrm{~nm}$ and used for calculations.

LC-MS/MS analysis. For LC-MS/MS analysis, green bands of lpCN-PAGE were cut into slices. The proteins in each gel slice were in-gel digested with trypsin and identified by $\mathrm{LC}-\mathrm{MS} / \mathrm{MS}^{28}$. For the label-free estimate of relative protein abundances in each band from LC-MS/MS data, we used the exponentially modified protein abundance index method ${ }^{29}$.

\section{References}

1. Hill, R. \& Bendall, F. Function of the two cytochrome components in chloroplasts: a working hypothesis. Nature 186, 136-137 (1960).

2. Liu, H. et al. Phycobilisomes supply excitations to both photosystems in a megacomplex in cyanobacteria. Science 342, 1104-1107 (2013).

3. van der Weij-de Wit, C. D., Ihalainen, J. A., van Grondelle, R. \& Dekker, J. P. Excitation energy transfer in native and unstacked thylakoid membranes studied by low temperature and ultrafast fluorescence spectroscopy. Photosynth. Res. 93, 173-182 (2007).

4. Iwai, M., Yokono, M. \& Nakano, A. Visualizing structural dynamics of thylakoid membranes. Sci. Rep. 4, 3768-3773 (2014).

5. Suorsa, M. et al. Dark-adapted spinach thylakoid protein heterogeneity offers insights into the photosystem II repair cycle. Biochim. Biophys. Acta 1837, 1463-1471 (2013).

6. Järvi, S., Suorsa, M., Paakkarinen, V. \& Aro, E. M. Optimized native gel systems for separation of thylakoid protein complexes: novel super- and megacomplexes. Biochem. J. 439, 207-214 (2011).

7. Booth, P. et al. Thermodynamic properties of D1/D2/cytochrome $b-559$ reaction centres investigated by time-resolved fluorescence measurements. Biochim. Biophys. Acta 1016, 141-152 (1990).

8. Mimuro, M. et al. Delayed fluorescence observed in the nanosecond time region at $77 \mathrm{~K}$ originates directly from the photosystem II reaction center. Biochim. Biophys. Acta 1767, 327-334 (2007).

9. Ilioaia, C., Johnson, M. P., Horton, P. \& Ruban, A. V. Induction of efficient energy dissipation in the isolated light-harvesting complex of photosystem II in the absence of protein aggregation. J. Biol. Chem. 283, 29505-29512 (2008).

10. Pålsson, L.-O., Tjus, S. E., Andersson, B. \& Gillbro, T. Energy transfer in photosystem I. Time resolved fluorescence of the native photosystem I complex and its core complex. Chem. Phys. 194, 291-302 (1995).

11. Mimuro, M., Yokono, M. \& Akimoto, S. Variations in photosystem I properties in the primordial cyanobacterium Gloeobacter violaceus PCC 7421. Photochem. Photobiol. 86, 62-69 (2010).

12. Yokono, M., Murakami, A. \& Akimoto, S. Excitation energy transfer between photosystem II and photosystem I in red algae: larger amounts of phycobilisome enhance spillover. Biochim. Biophys. Acta 1807, 847-853 (2011).

13. Galka, P. et al. Functional analyses of the plant photosystem I-light-harvesting complex II supercomplex reveal that light-harvesting complex II loosely bound to photosystem II is a very efficient antenna for photosystem I in state II. Plant Cell 24, 2963-2978 (2012).

14. Passarini, F., Wientjes, E., van Amerongen, H. \& Croce, R. Photosystem I lightharvesting complex Lhca4 adopts multiple conformations: red forms and excited-state quenching are mutually exclusive. Biochim. Biophys. Acta 1797, 501-508 (2010).

15. Jennings, R. C., Zucchelli, G. \& Santabarbara, S. Photochemical trapping heterogeneity as a function of wavelength, in plant photosystem I (PSI-LHCI) Biochim. Biophys. Acta 1827, 779-785 (2013). 
16. Wientjes, E., van Stokkum, I. H. M., van Amerongen, H. \& Croce, R. The role of the individual Lhcas in photosystem I excitation energy trapping. Biophys. J. 101, 745-754 (2011).

17. Knoetzel, J., Svendsen, I. \& Simpson, D. J. Identification of the photosystem I antenna polypeptides in barley. Eur. J. Biochem. 206, 209-215 (1992).

18. Kato, Y. \& Sakamoto, W. Phosphorylation of photosystem II core proteins prevents undesirable cleavage of $\mathrm{D} 1$ and contributes to the fine-tuned repair of photosystem II. Plant J. 79, 312-321 (2014).

19. Grieco, M., Tikkanen, M., Paakkarinen, V., Kangasjärvi, S. \& Aro, E. M. Steadystate phosphorylation of light-harvesting complex II proteins preserves Photosystem I under fluctuating white light. Plant Physiol. 160, 1896-1910 (2012).

20. Kowalczyk, N. et al. Photosynthesis in Chondrus crispus: the contribution of energy spill-over in the regulation of excitonic flux. Biochim. Biophys. Acta 1827, 834-842 (2013).

21. Takabayashi, A. et al. The oligomeric states of the photosystems and the light-harvesting complexes in the chl $b$-less mutant. Plant Cell Physiol. 52, 2103-2114 (2011).

22. Yokono, M. et al. Alterations in photosynthetic pigments and amino acid composition of D1 protein change energy distribution in photosystem II. Biochim. Biophys. Acta 1817, 754-759 (2012).

23. Yokono, M., Akimoto, S. \& Tanaka, A. Seasonal changes of excitation energy transfer and thylakoid stacking in the evergreen tree Taxus cuspidata: how does it divert excess energy from photosynthetic reaction center? Biochim. Biophys. Acta 1777, 379-387 (2008).

24. Ohashi, K., Murakami, A., Tanaka, A., Tsuji, H. \& Fujita, Y. Developmental changes in amounts of thylakoid components in plastids of barley leaves. Plant Cell Physiol. 33, 371-377 (1992).

25. Hiyama, T. \& Ke, B. Difference spectra and extinction coefficients of P700. Biochim. Biophys. Acta 267, 160-171 (1972).

26. Kaminskaya, O., Kern, J., Shuvalov, V. A. \& Renger, G. Extinction coefficients of cytochromes b559 and c550 of Thermosynechococcus elongatus and Cyt b559/ PS II stoichiometry of higher plants. Biochim. Biophys. Acta 1708, 333-341 (2005).

27. Porra, R., Thompson, W. \& Kriedemann, P. Determination of accurate extinction coefficients and simultaneous equations for assaying chlorophylls $a$ and $b$ extracted with four different solvents: verification of the concentration of chlorophyll standards by atomic absorption spectroscopy. Biochim. Biophys. Acta 975, 384-394 (1989).
28. Takabayashi, A. et al. Protein co-migration database (PCoM-DB) for Arabidopsis thylakoids and Synechocystis cells. Springerplus 2, 1-10 (2013).

29. Ishihama, Y. et al. Exponentially modified protein abundance index (emPAI) for estimation of absolute protein amount in proteomics by the number of sequenced peptides per protein. Mol. Cell Proteomics 4, 1265-1272 (2005).

30. Vizcaíno, J. et al. ProteomeXchange provides globally co-ordinated proteomics data submission and dissemination. Nat. Biotechnol. 30, 223-226 (2014).

\section{Acknowledgements}

We gratefully thank Kayo Nakamura, Saeka Takabayashi, Yukako Kato and Yasuhiro Kasahara for LC-MS/MS measurement and analyses. This work was supported in part by Special Coordination Funds for promoting Science and Technology, Creation of Innovation Centers for Advanced Interdisciplinary Research Areas (Innovative Bioproduction, Kobe), Japan.

\section{Author contributions}

M.Y. designed and performed the research. A. Takabayashi arranged the mutants and native-PAGE systems, and performed LC-MS/MS analyses. A. Tanaka prepared purified standard samples and performed absorption spectroscopy. S.A. developed devices for the measurement of time-resolved fluorescence. M.Y. analysed the data and wrote the paper. All authors reviewed and discussed the manuscript.

\section{Additional information}

Accession codes: the mass spectrometry proteomics data have been deposited to the ProteomeXchange Consortium ${ }^{30}$ via the PRIDE partner repository with the data set identifier PXD001803.

Supplementary Information accompanies this paper at http://www.nature.com/ naturecommunications

Competing financial interests: The authors declare no competing financial interests.

Reprints and permission information is available online at http://npg.nature.com/ reprintsandpermissions/

How to cite this article: Yokono, M. et al. A megacomplex composed of both photosystem reaction centres in higher plants. Nat. Commun. 6:6675 doi: 10.1038/ncomms7675 (2015). 